\title{
A Simulated Study on Performance Evaluation of a Communication Network Model with DSR Protocol using ViSim
}

\author{
U. Nanaji ${ }^{1}$, Nakka Thirupathi Rao ${ }^{1}$, Ch. Raj Kumar ${ }^{1}$, \\ Debnath Bhattacharyya ${ }^{1}$ and Hye-jin Kim ${ }^{2}$ \\ ${ }^{1}$ Department of Computer Science and Engineering, Vignan's Institute of \\ Information Technology, Visakhapatnam-530049, AP, India \\ ${ }^{2}$ Sungshin Women's University, ${ }^{2}$ Bomun-ro 34da-gil, Seongbuk-gu, Seoul, Korea \\ nakkathiru@gmail.com,debnathb@gmail.com, hyejinaa@daum.net, \\ (Corresponding Author)
}

\begin{abstract}
The investigation of the impact of network size and simulation area on DSR reactive routing protocol with mobility of nodes was studied and analyzed in this paper. The protocol was simulated using virtual hosts on a discrete-event network simulator: ViSim. The protocol was run on a simulation setup of 3,10 and 20 nodes simulation areas. A performance study of the protocols is done based on available parameters which are Qos metrics viz. end-to-end delay, throughput and other network performance metrics. The analysis of the performance of the protocol at three different scenarios. The results were represented and discussed in the results section in detail.
\end{abstract}

Keywords: DSR, ViSim, Quality of Service (QoS) metrics, Throughput, Routing Bytes, Routing Load

\section{Introduction}

In MANETs (Mobile Ad-hoc Networks) performance is sensitive to mobility, scalability and traffic load etc. Examining different routing protocols performance in dynamic environment plays a vital role in efficient routing. Here in my default parameters of DSR routing protocol in MANET's for finding the impact in Qos Metrics which are playing a crucial role in the performance of an infrastructure less network. And also finding the performance of different configurations in routing protocols of MANETs along with varying default various parameters results such as Qos parameters (throughput, delay etc.).

The main aim of this thesis is to analyze the performance of DSR routing protocol under different scenarios. In MANETs based on various parameters and also to investigate the impact of network area (i.e., simulation area) and VBR variable bit rate and CBR constant bit rate on the performance of reactive routing protocol. And finding the impact by changing the parameters of DSR routing protocol in Ad-hoc networks.

In this paper, the main contribution is to analyze the performance of reactive routing protocol in ad-hoc networks under different scenarios based on different parameters by using and ViSim simulator. Using ViSim simulator by varying Qos parameters and analyzing the performance of DSR reactive routing protocol.

\subsection{Research Findings}

To analyze various parameters of Qos, the analysis of different parameters of MAC layer such as dropped PkNotforUs:sum, PassedUpPk:sum, RcvdPkFromHL:sumRcvdPkFromLL: sum, SentDownPk:sum under shortest path and 
static configurations by varying bitrate using ViSim. We also extended our analysis various parameters of Qos like throughput, delay, load using ViSim.

- The impact of transmission bit rate.

- The impact by varying network size and simulation area.

- The impact by varying Threshold under TTL Parameter.

\subsection{Mobile Ad-Hoc Networks}

Mobile Adhoc networks are the wireless networks which were mostly used in the recent times in the real time world applications. The nodes in the mobile networks are changes from time to time with respect to their position of the nodes in the actual architecture of the network. As the nodes positions changes, the topology of the network changes from time to time. As the topology of the network changes from time to time, the performance of the network influences a lot and the effect was followed to the other nodes in the same network and other nodes which were connected to the same networks. The ad hoc networks are the self-configuring networks which will change the positions of the networks and their locations based on the requirements of the network and their performance. The mobile devices are allowed freely to move chaotically and consolidate themselves subjectively.

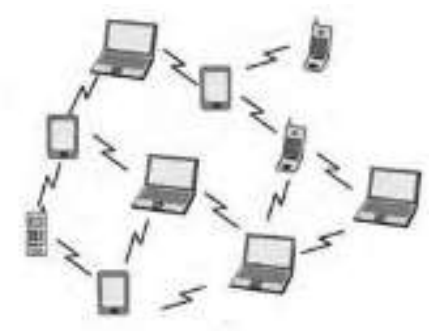

Figure 1. Mobile Ad-Hoc Network

\section{Routing Protocols in MANET'S}

\subsection{Problems with Routing in MANET's}

i. Wireless Links: The most important point to be considered whenever we are using a wireless network especially all the nodes in the sensor network are working under wireless mode. As the nodes are connected in the mode of a wireless mode, all the nodes in a wireless network are able to easily be attacked by other attackers in the network. Wired networks cannot need to be conducted or to be issued as the nodes do not require the physical connection to access the data from the nodes and the attacks on the nodes in the network. These wireless networks will have very less bandwidth when compared to the other set of networks like the wired networks. Hence, for using the wireless networks the bandwidth should need to be minimum.

ii. Dynamic Topology: The nodes in the mobile Adhoc networks are in the dynamic in nature in terms of the topology and their working. The topology of the network and the nodes which were connected in the network might change from time to time. As the nodes in the wireless network changes from time to time, the topology of the network changes and the amount of the load on the network and behavior of the nodes in the network and the performance of the network also changes as a result of the effects that were being taken place. As the topology and the position of the nodes in the network changes from time to time, the attacks on the nodes might also be increased in a good number. 
iii. Cooperativeness: The behavior and all the nodes present and working in the wireless networks are easily and heavily cooperative with each other. But, in reality several factors will influence the cooperativeness between the various nodes. Due to the presence of various set of nodes with various loads ion the each node and the topology of the nodes that were connected in the networks might have a great impact on the togetherness between $\mathrm{t}$ all the nodes in the network. Malicious attacks on each node in the network can be easily possible. The malicious attacker or the node in the network can become one of the most important agent for routing 8in the network and the various operations in the network can be disturbed by various nodes and other set of operations in the network.

iv. Lack of a Clear Line of Defense: The defense system for identifying the malicious nodes and the effect of these nodes can be prevented at earlier times in some cases and in some cases it is not possible also. The direction from which the malicious attacks or the attacks on the nodes in the network cannot be predicted at an earlier time in some cases it not possible also.

v. Limited Resources: The other important consideration to considered was the availability of the resources to the users and for also to the systems. Several different types of devices were available in the network ranging from the devices like handheld devices like the mobiles, pagers and a other devices to the laptops and other devices. As these devices will have the various components to work with, the working condition and support to other devices may change from time to time. As the data was not be the same in all devices to store, the storing of data in these sorts of systems or the devices was not an easy task. The storage capacity of these devices might change from device to device and also from source to source.

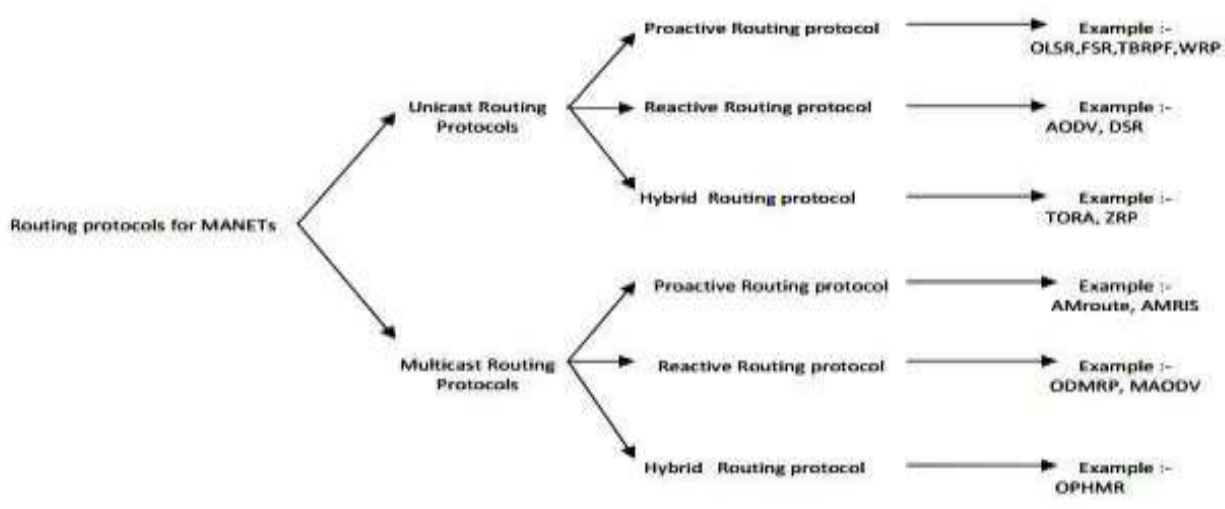

Figure 3. Classification of Routing Protocols for MANET's

\subsection{Qos Metrics}

i. Load: Total number of packets received or transmitted in a channel of a network is called as load.

ii. Average end-to-end delay: It is defined as the average amount of time taken for a packet to travel from source to the destination in a network.

Avg. EED=Total EED/No. of Packets

iii. Throughput: It is defined as the amount of data that can be transferred from sender to receiver in a given amount of time in a network.

Throughput $=$ Number of packets sent/ Time Taken 


\subsection{Routing load}

Routing load: The routing load in the network can be calculated as the total number of routing packets that are sent within the network divided by the total number of packets that are transmitted within the network to reach the destination.

\section{Simulation Tool Using ViSim}

ViSim is one of the mostly used simulators in the field of research and in the industry. The main reasons for the famous of this toll is due to its user friendly user interface so that the users can use easily and also can make the changes to the existing network combinations. Because of these facilities, the ViSim might be helpful for researchers, students, teachers in their respective research work, and for the expression of a variety of wireless network scenarios on computer screens and its related user output devices. The usage of this simulator had become the task of simulation for various devices had made the things and the simulator models become very famous and very easy. The interest for the users for utilizing this simulator had grown a lot in the customers and users for this simulator in these days.

When compared to the other set of simulators including the writing command interfaces, the task were become hard for the users. But, with the inclusion and usage of these simulator models, the representation and creation of various concepts and certain conditions for the development of networks by using this simulator had become very famous and can be used by most of the users in the market today. ViSim is not a simulation engine, somewhat it calls ns-2 simulation models in the backdrop and makes the assignment trouble-free for the end users to envisage the simulation in a Windows surroundings. Performance Evaluation of the network with DSR protocol using ViSim was shown in the following figures and it was executed various scenarios of the network and its related components. Using the present simulation tool, the performances of several Mobile Ad-hoc Network (MANET) routing protocols were analyzed and noted as graphical representations and tabular formats were represented in the following graphs.

\subsection{Implementation of ViSim Scenario}

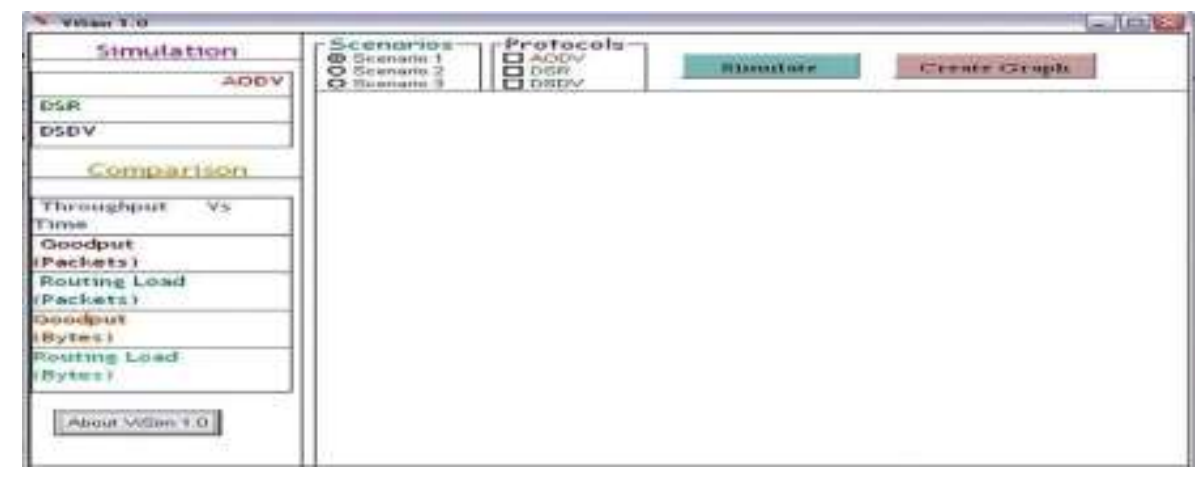

Figure 4. Selection of Scenarios in the User Interface of the Simulator

In the above figure, the selection of various and other related things can be selected by using or creating the different scenarios with various constraints. Various scenarios with the combination of several protocols in the network with various network performance metrics were selected from the above given figure. 


\subsection{Functional Examples}

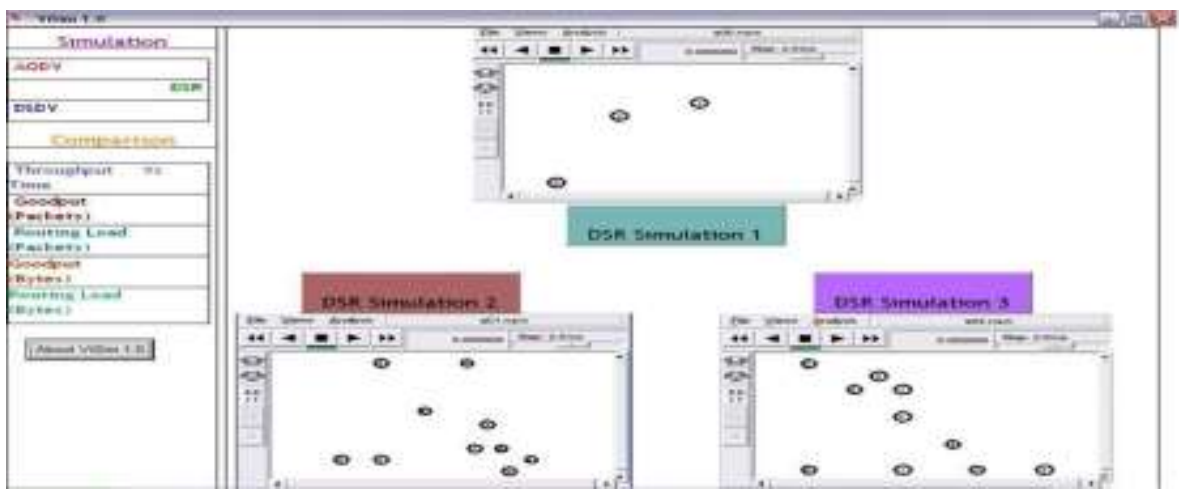

Figure 5. Creation of Three Scenarios in the User Interface of the Simulator

In the above figure, the three sets of nodes were selected and a three network models with three scenarios were created by using the simulator with the combination of the DSR protocol. All the three network models were working with the help of the DSR protocol.

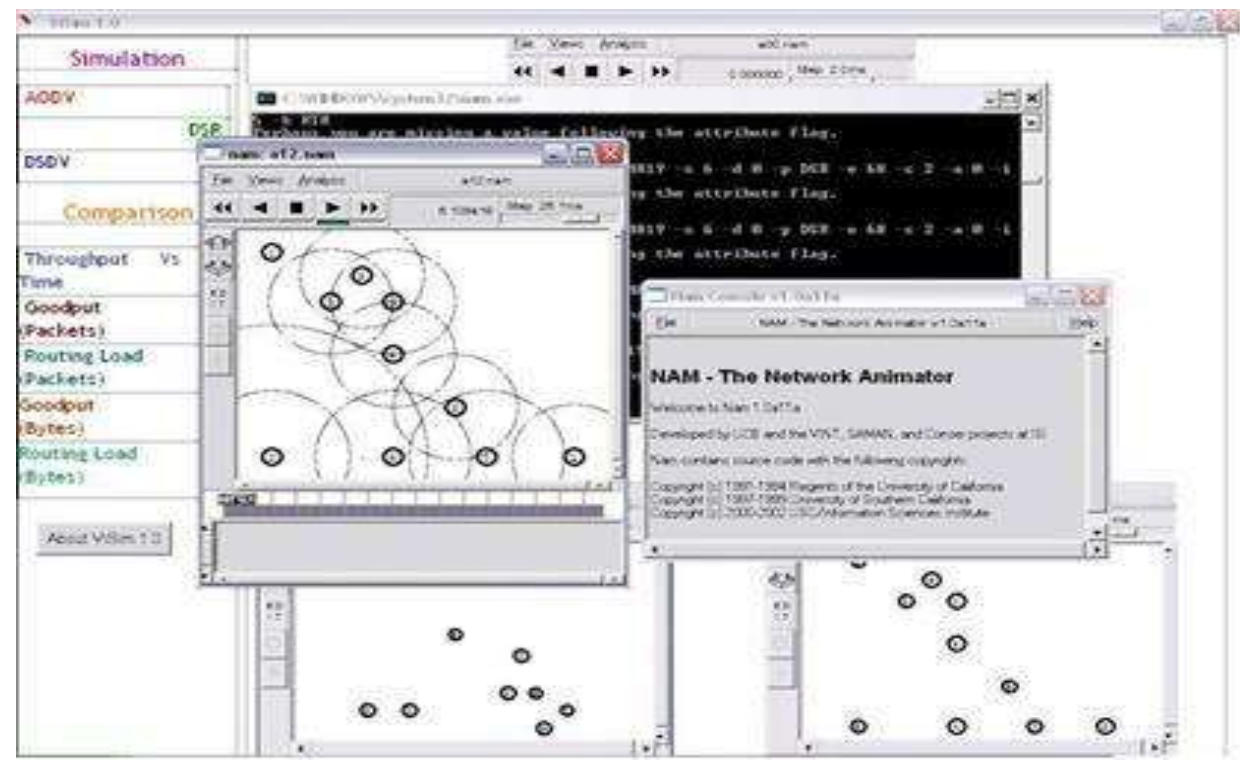

Figure 6. The Output after Choosing DSR Simulation 3

From the above figure, the creation of several nodes in the assumed network with various loads considerations and their configurations with their loads of input and output of the nodes were also discussed so far. The figure gives an brief idea of creating the nods and their programming model for the working and executing process of the network model was also to be considered and implemented in the following figures. 


\section{Simulation of the 3 Scenarios}

\subsection{Scenario-1(3 Nodes)}

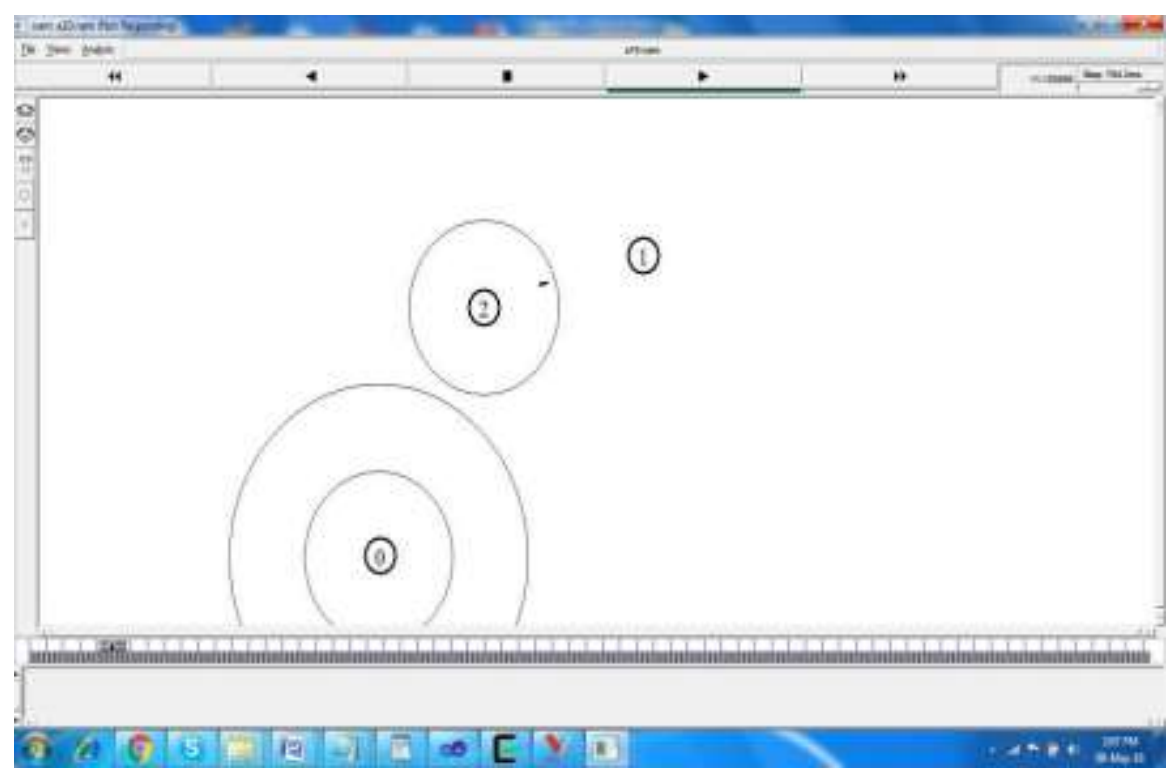

Figure 7. The Creation of Three Nodes in the Scenario 1

In the above figure, the creation of the three nodes in the simulator model was displayed.

\subsection{Scenario-2(10 Nodes)}

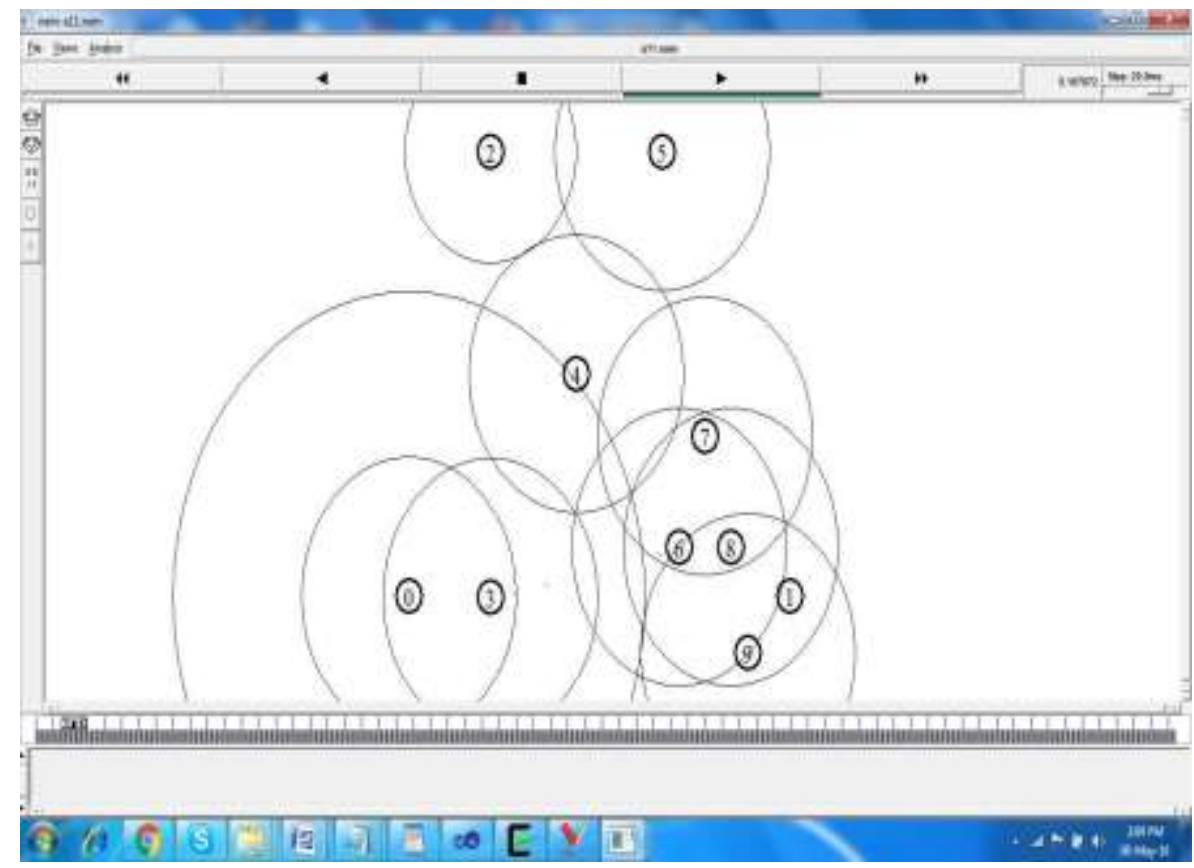

Figure 8. The Creation of Ten Nodes in the Scenario 2

In the above figure, the simulator was selected such that to create a set of ten nodes in the wireless network as the scenario 2 . 


\subsection{Scenario-3(20 Nodes)}

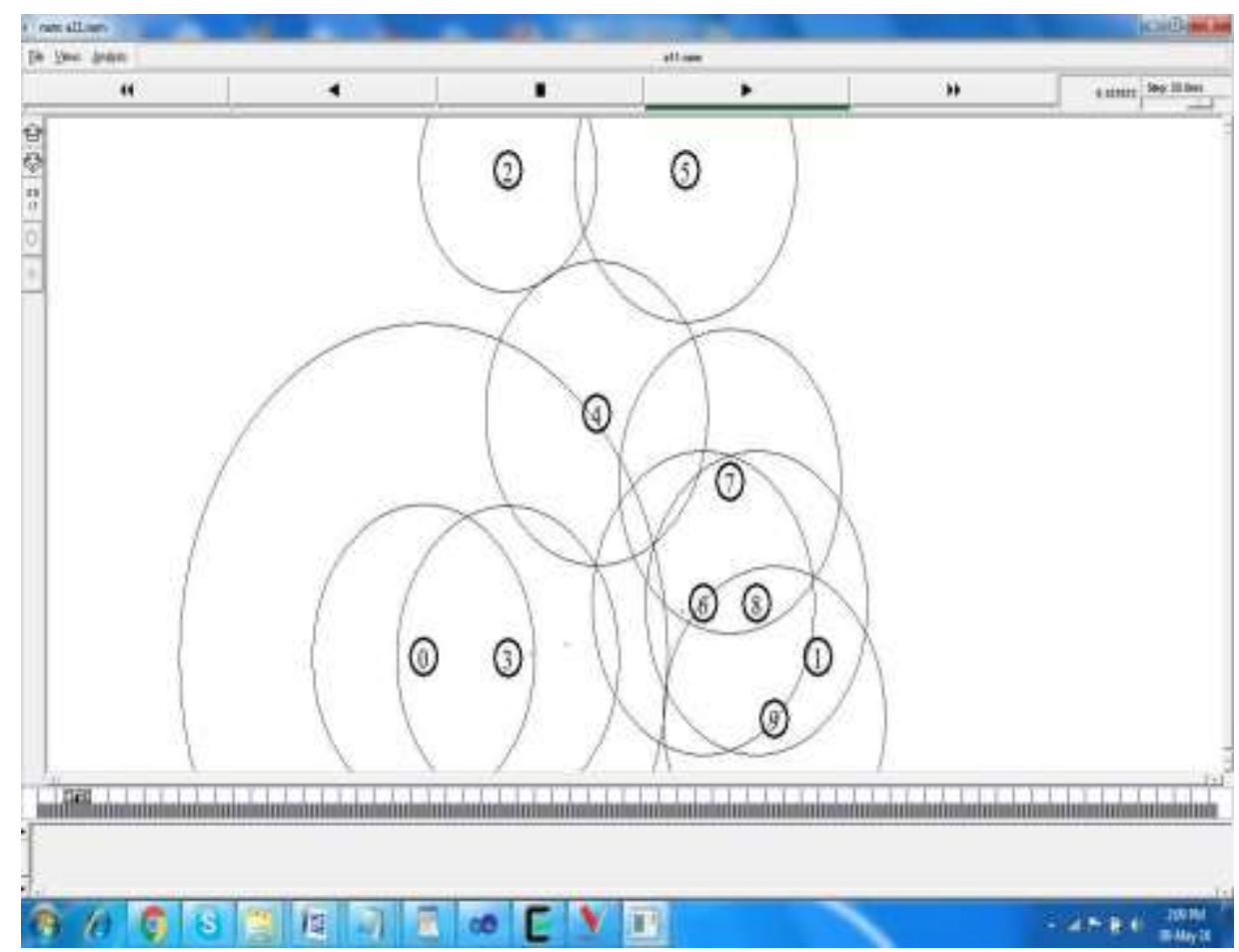

Figure 9. The Creation of Twenty Nodes in the Scenario 3

In the above figure, the simulator was selected such that to create a set of twenty nodes in the wireless network as the scenario 3.

\subsection{Throughput of DSR at 3,10 and 20 Nodes}

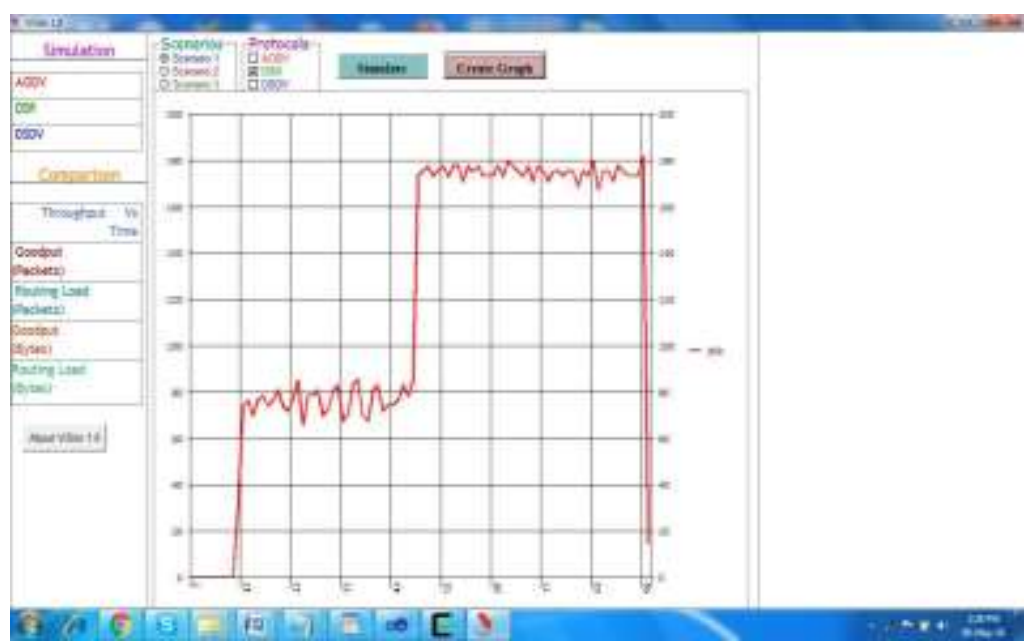

Figure 10. The Throughput of Three Nodes in rhe Scenario 1

In the above figure, the simulator was selected such that the throughput of the network with three set nodes at scenario 1 was studied and represented. 


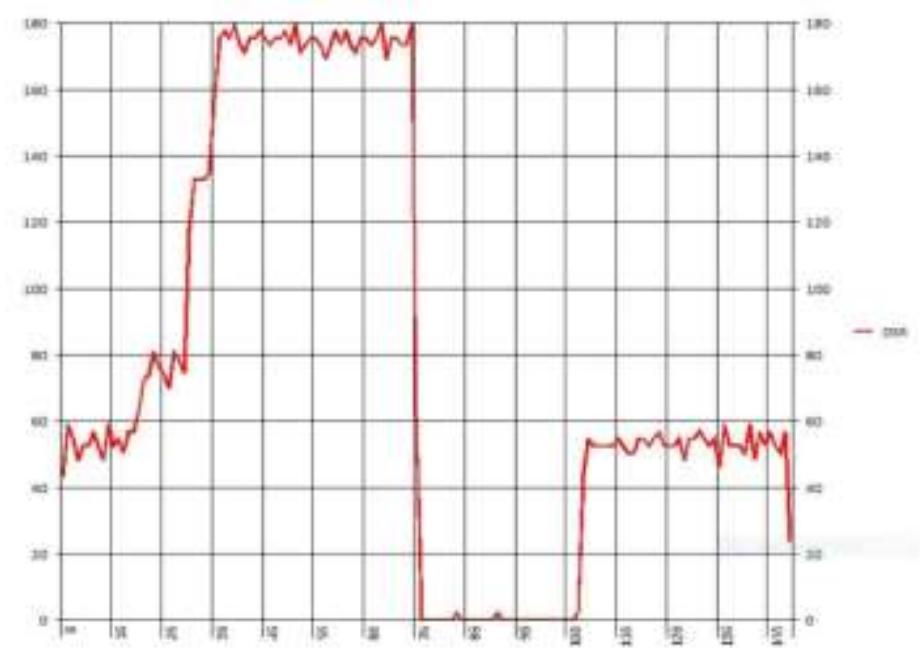

Figure 11. The Throughput of Ten Nodes in the Scenario 2

In the above figure, the simulator was selected such that the throughput of the network with ten nodes at scenario 2 was studied and represented.

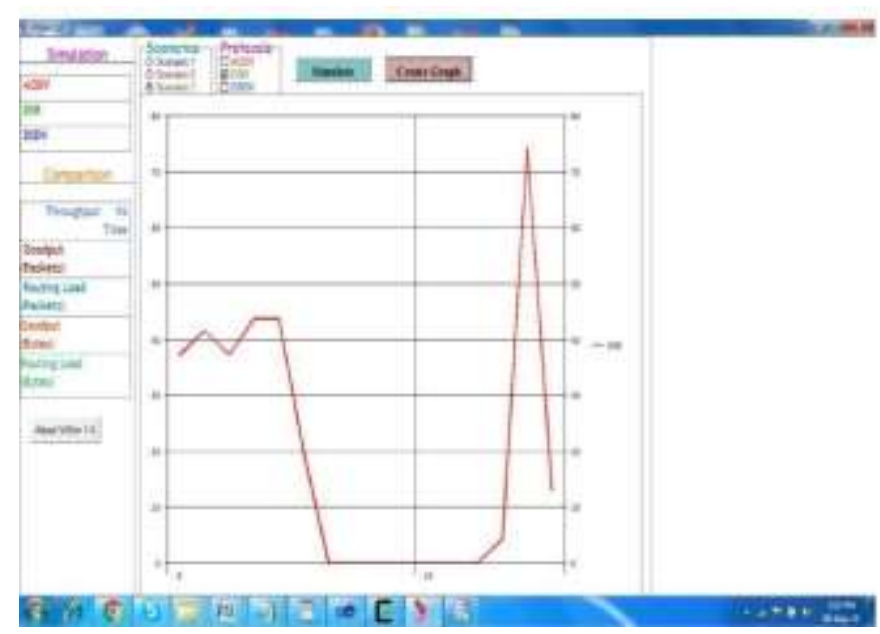

Figure 12. The Throughput of Twenty Nodes in the Scenario 3

In the above figure, the simulator was selected such that the throughput of the network with twenty nodes at scenario 3 was studied and represented. 


\subsection{Routing Load Packets at 3, 10 and 20 Nodes}

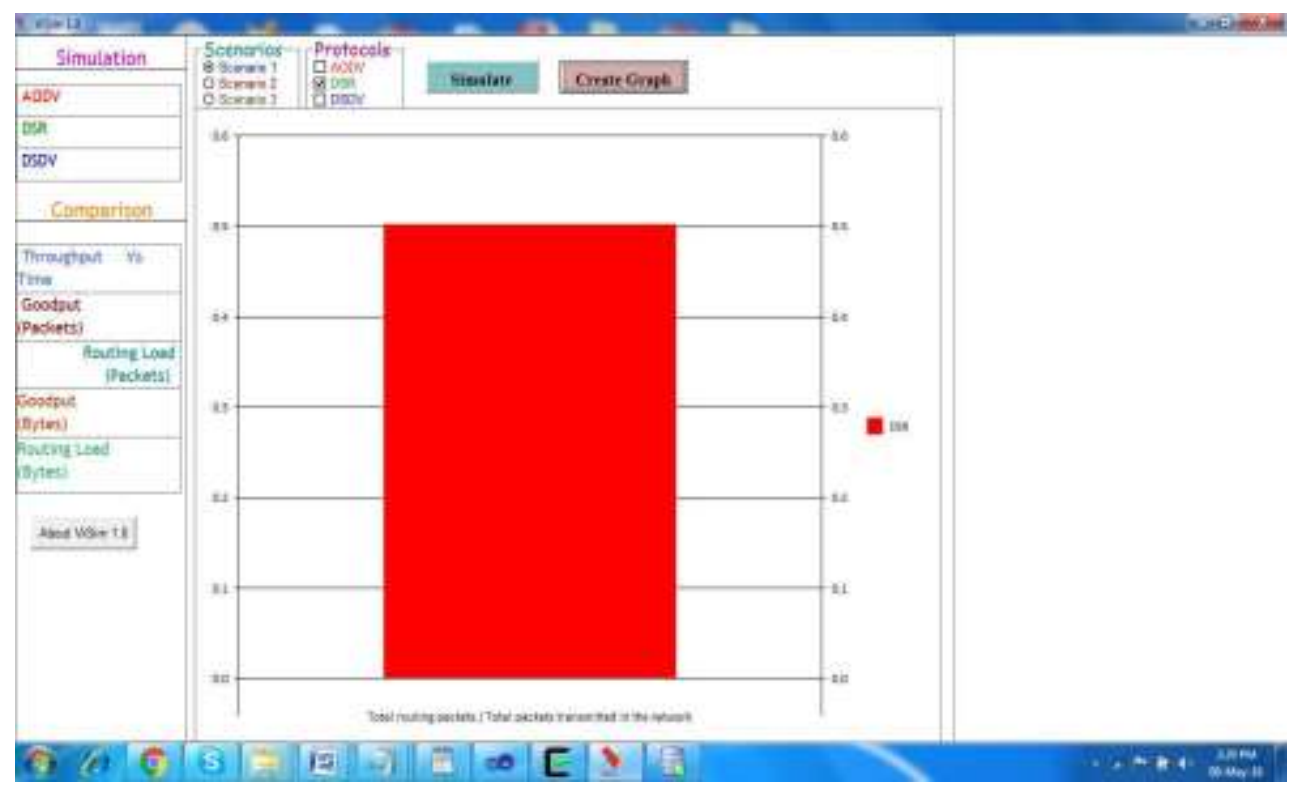

Figure 13. The Routing Load on the Packets Three Nodes in the Scenario 1

In the above figure, the simulator was selected such that the routing load on the network was analyzed with the help of the graphical representation in the above Figure 13. The number of nodes that were selected is the three nodes in the network.

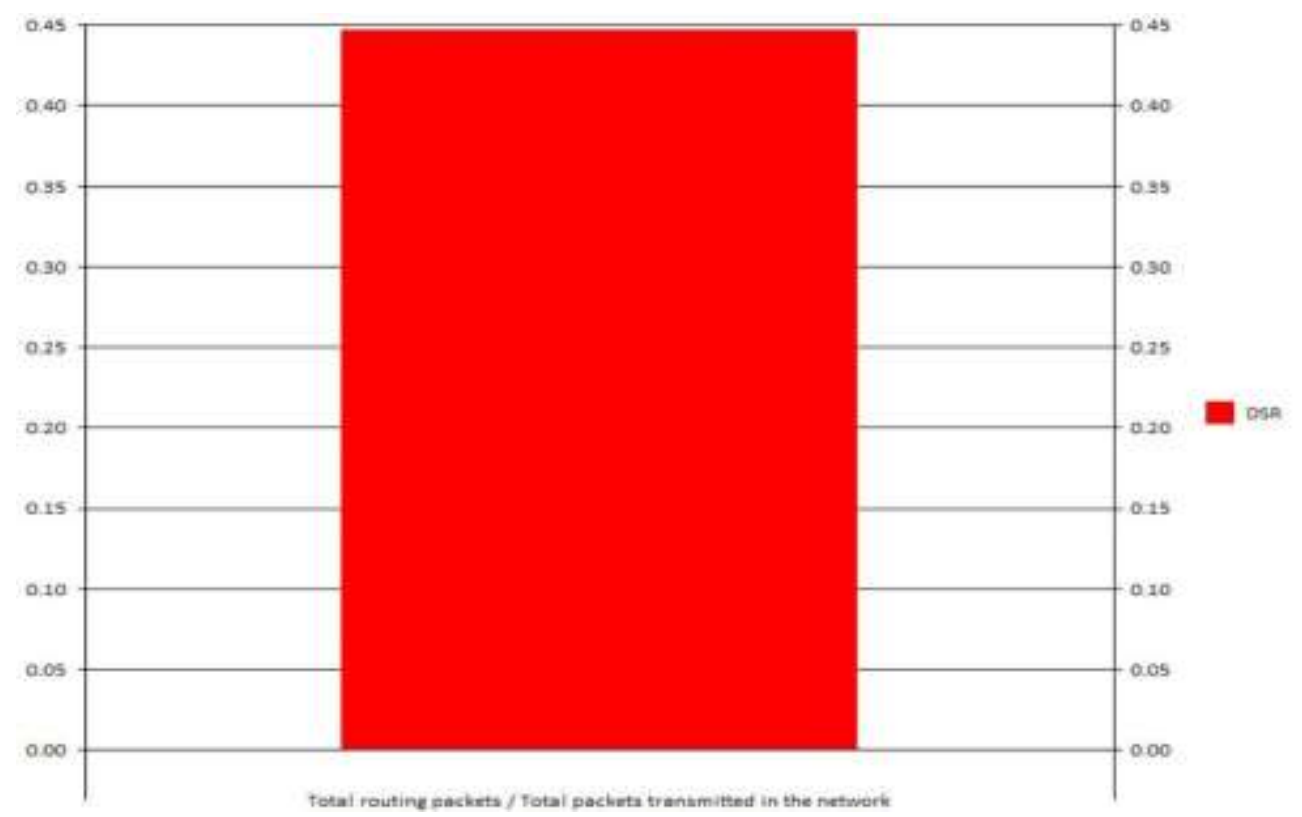

Figure 14. The Routing Load on the Packets Ten Nodes in the Scenario 2

In the above figure, the simulator was selected such that the routing load on the network was analyzed with the help of the graphical representation in the above Figure 14. The number of nodes that were selected is the ten nodes in the network. 


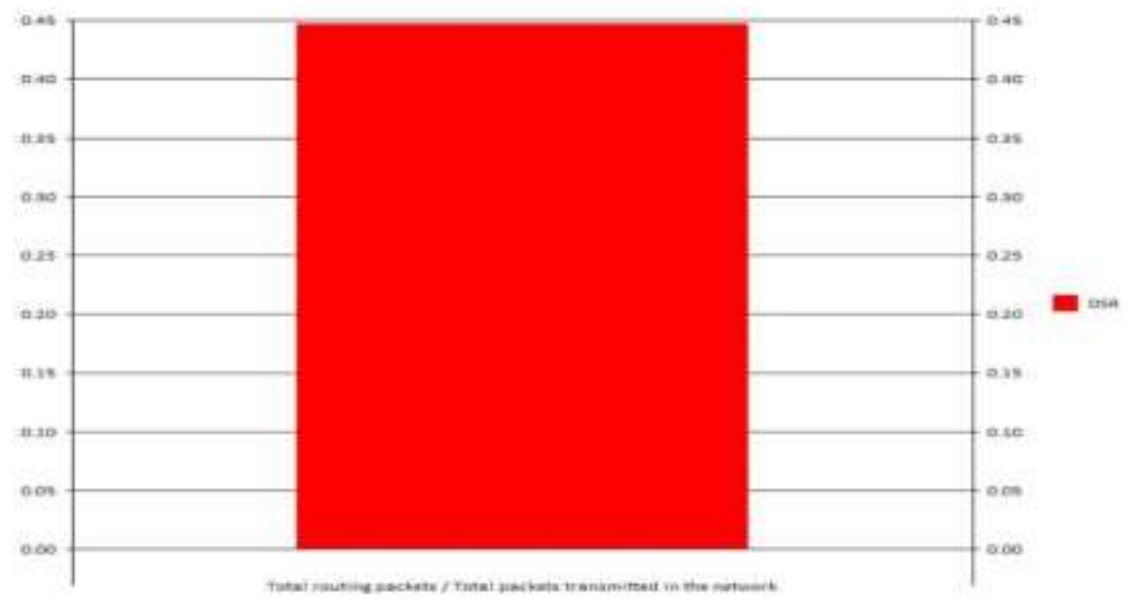

Figure 15. The Routing Load in the Packets Twenty Nodes in the Scenario 3

In the above figure, the simulator was selected such that the routing load on the network was analyzed with the help of the graphical representation in the above Figure 15. The number of nodes that were selected is the twenty nodes in the network.

\subsection{Routing Load Bytes at 3, 10 and 20 Nodes}

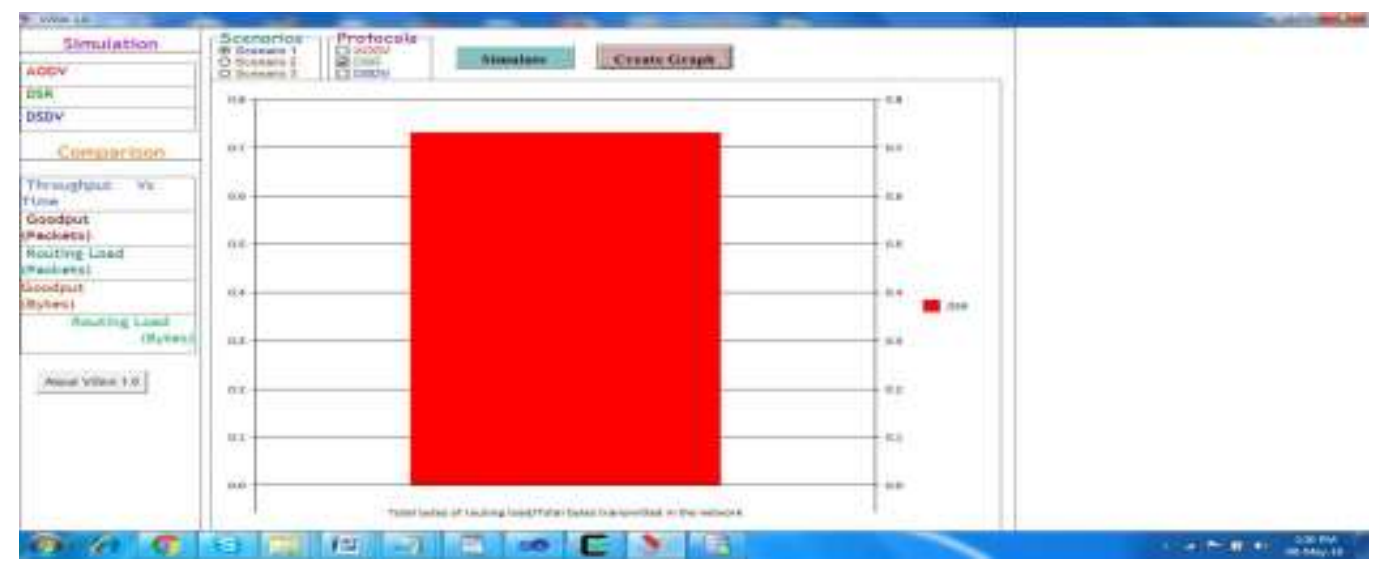

Figure 16. The Routing Load on the Packets Three Nodes in the Scenario 1

In the above figure, the simulator was selected such that the routing load in terms of bytes on the network was analyzed with the help of the graphical representation in the above Figure 16. The number of nodes that were selected is the twenty nodes in the network. 


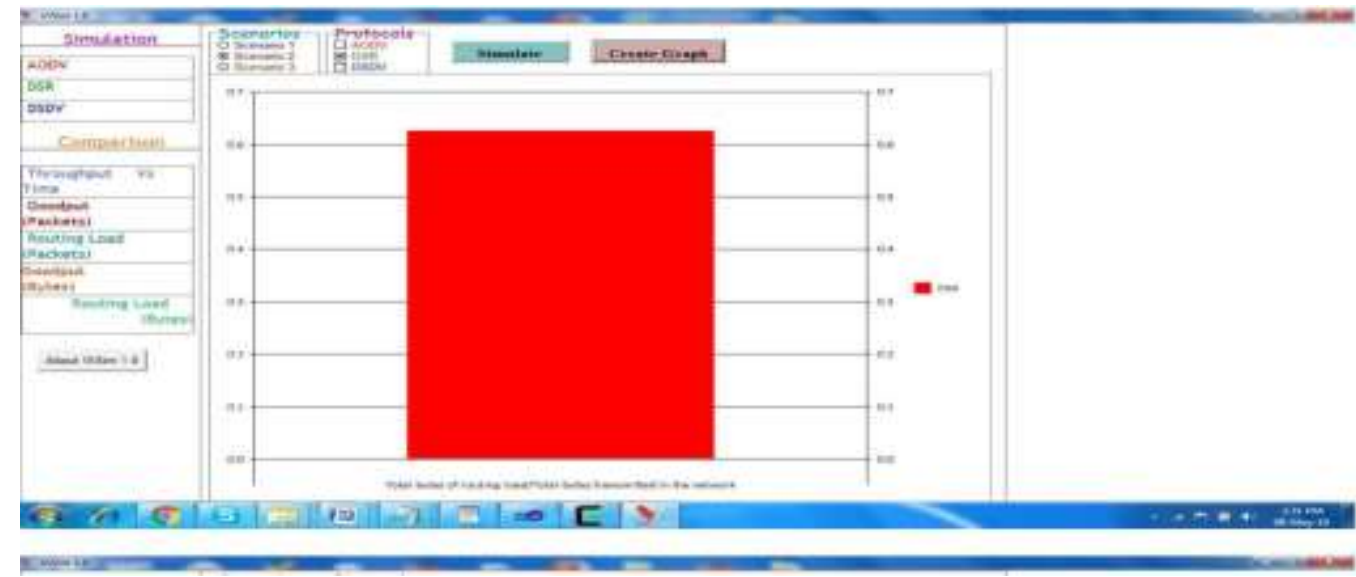

Figure 17. The Routing Load on the Packets Ten Nodes in the Scenario 2

In the above figure, the simulator was selected such that the routing load in terms of bytes on the network was analyzed with the help of the graphical representation in the above Figure 17. The number of nodes that were selected is the ten nodes in the network.

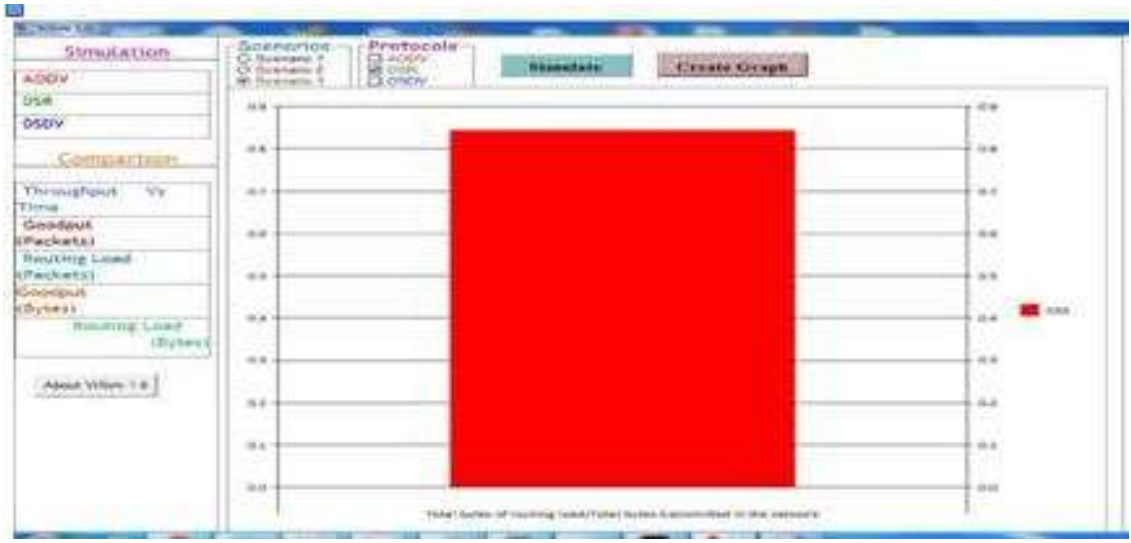

Figure 18. The Routing Load on the Packets Twenty Nodes in the Scenario 3

In the above figure, the simulator was selected such that the routing load in terms of bytes on the network was analyzed with the help of the graphical representation in the above Figure 18. The number of nodes that were selected is the twenty nodes in the network.

\section{Conclusion}

In this paper, the performance of the wirelesses sensor network with numerous figures of nodes in the network at various combinations called as various scenarios were discussed and presented. The simulation tool ViSim was used successfully and total performance of the network at various conditions and the performance metrics of the networks were analyzed with the help of graphical representations. The various performance metrics of the communication networks like the throughput of the nodes in the network at various levels like the three nodes in the network, ten nodes in the network and twenty nodes in the network. The results and the performance were successfully represented by using the graphical representation. Several users trade with ns-2 simulations expression problem in set up Linux or other systems and environment. The use of ActiveTcl with a graphical ViSim interface was very much helpful to the users like the researchers and the people from both software and hardware industries. The 
performance of the network with the use of DSR protocol in the network for data transmissions was studied successfully with the combination of three set of nodes in each scenario. Three nodes, ten nodes and twenty nodes were considered in each case and the performance was analyzed with the help of graphical representation. There were several fluctuations in the curve of the throughput of the network which could be a problem in the case of performance of the network. The fluctuations in the throughput of a network were undesirable for any customers or user whoever is using these setoff nodes at various combinations.

\section{References}

[1] B. Wu, "Simulation Based Performance Analyses on RIPv2, EIGRP, and OSPF Using OPNET", (2011).

[2] D. Xu. "OSPF, EIGRP, and RIP performance analysis based on OPNET", Internet: www.sfu.ca/ donx, (2013).

[3] J. Varsalone, "In Cisco CCNA/CCENT Exam 640-802, 640-822, 640-816 preparation kit [electronic resource]: with Cisco router simulations", Rockland, Mass. : Syngress ; Oxford: Elsevier Science, (2009).

[4] U.D. Black, "In IP routing protocols: RIP, OSPF, BGP, PNNI, and Cisco routing protocols", Upper Saddle River, NJ: Prentice Hall, (2000).

[5] M. K. Denko, "In Mobile opportunistic networks: architectures, protocols and applications", Boca Raton: CRC Press, (2011).

[6] D. Medhi and K. Ramasamy, "In Network routing: algorithms, protocols, and architectures", Amsterdam; Boston: Elsevier: Morgan Kaufmann Publishers, (2007).

[7] P. E. B. Royer and S. Dass, "Ad hoc On-Demand Distance Vector (AODV) Routing", Memo of Network Working Group, available online: http://www.ietf.org/rfc/rfc3561.txt, (2003).

[8] P. E. B. Royer, S. Dass and I. Chakeres, "AODV”, available online: http://moment.cs.ucsb.edu/AODV/ aodv.html, (2010).

[9] H. P. Wang and L. Cui, “An Enhanced AODV for Mobile Ad Hoc Network”, vol. 7. 